\title{
План контроля дымовых газов енергопредприятий с учетом нарушений стационарности выбросов
}

\author{
Н.А. Любимова, В.К. Пузик, Л.М. Пузик \\ Харковский национальный технический университет сельського хозяйства \\ имени Петра Василенко (м.Харков, Украина) \\ email:1 nina.lioubimova@gmail.com , 2 kysmish@gmail.com ,3 Iudapusik@gmail.com, \\ ORCID: ${ }^{1}$ 0000-0001-8964-7326 , ${ }^{2}$ 0000-0001-5028-9461, ${ }^{3}$ 0000-0002-5465-2771
}

\begin{abstract}
Данное исследование посвящено планированию и прогнозированию контроля дымовых газов крупных энергоемких предприятий. Появление случайных технологических нарушений провоцирует случайность выбросов, нарушение норм природопользования, экономические санкции и штрафы, ведет к деградации окружающей среды. Разработана математическая модель планирования и прогнозирования экстремальных выбросов в случайных процессах загрязнения дымовых газов энергетических предприятий с коррекцией на нестационарность процессов. Описана процедура параметрического тестирования многокомпонентного процесса загрязнения при многопараметрическом контроле с учетом его экстраполированных значений. Использование скорректированной, в сторону увеличения математического ожидания, модели экстраполяции 2-го порядка, снижает риск контроля 2-го порядка $\beta$, при параметрическом тестировании результатов многомерной экстраполяции. Уменьшение риска контроля $\beta$ повышает мощность правила принятия решений и делает применение описанной скорректированной процедуры экстраполяции статистически обоснованным и более предпочтительным по отношению к другим процедурам экстраполяции. Целесообразно при планировании и прогнозировании контроля выбросов и сбросов энергоемких предприятий использовать тестирование с учетом правила принятия решений на основе разработанного критерия, что обеспечивает заданную достоверность. Оценка стационарности и спектральных особенностей исследуемых процессов загрязнения позволит уменьшить априорную неопределенность и даст возможность усовершенствования процедуры контроля дымовых газов. Выбор правила принятия решений на основе критерия, обеспечивающего заданную достоверность контроля, позволит исследовать корреляционную связность контролируемого процесса и разработать статистически обоснованный метод выбора интервала его дискретизации. Данный подход гарантирует минимизацию тех его рисков, которые определяют уровень экономических потерь при появлении нарушений стационарности. Предложенная модель контроля может быть использована при экологическом мониторинге выбросов в атмосферу энергоемких предприятий.
\end{abstract}

Ключевые слова: экология, модель, контроль, прогнозирование, многокомпонентное загрязнение

Постановка проблемы. Повышение достоверности контроля объектов с динамическими свойствами - это проблема получения избыточной информации, содержащейся в случайных, обычно нестационарных по математическому ожиданию, измерительных сигналах.

Такую избыточность могут дать методы обработки сигналов, учитывающие их связность и корреляционные свойства. При высокой связности, дополнительную информацию могут дать процедуры экстраполяции сигналов, однако, такая информация может быть утрачена из-за ошибок экстраполяции, возникающих при неадекватном выборе вероятностной модели сигнала или методически неправильных процедурах его обработки.

Анализ последних исследований и публикаций Статистические процедуры прогнозирования (экстраполяции) находят широкое применение в задачах контроля надежности энергетического оборудования [1], при прогнозировании работоспособности, отказов, остаточного технического ресурса [2-4]. В основном используют регрессионные модели экстраполяции 1-го порядка (по условному математическому ожиданию [5,6]), или более сложные модели 2-го и 3-го порядка (по одной [7] или двум $[7,8]$ точкам). Однако, все перечисленные модели базируются на симметричных законах распределения случайных сигналов и плохо работают, если нарушена их стационарность, вследствие искажений формы этих законов.

Цель статьи - показать возможность параметрической коррекции процедуры экстраполяций на несимметричность закона распределения случайного процесса и обосновать построение процедуры тестирования многомерного экстраполированного процесса при контроле его экстремальных выбросов. 
Общая модель прогнозирования. Широко используются модели экстраполяции не по математическому ожиданию (регрессии), а по одному, максимум по двум точкам $[7,8,9]$.

При этом достаточно знать нормированную автокорреляционную функцию $\rho(\tau)$ исследуемого процесса $x(t)$, его математическое ожидание $m$ и дисперсию $\sigma^{2}$. Известный алгоритм статистического прогноза по одной точке $[7,8]$, на интервале прогнозирования $\Theta$, формирует предсказанное значение $\hat{x}\left(t_{0}-m\right)$, отстоящее от предыдущего отсчета $x\left(t_{0}\right)$ на время $\Theta$, как

$$
\hat{x}\left(t_{0}+\Theta\right)=m+\rho(\Theta)\left[x\left(t_{0}\right)-m\right] . .
$$

Если $x\left(t_{0}+\Theta\right)$ - действительное значение процесса в момент времени $\left(t_{0}+\Theta\right)$, то ошибка прогноза равна

$$
\varepsilon\left(t_{0}+\Theta\right)=x\left(t_{0}+\Theta\right)-\hat{x}\left(t_{0}+\Theta\right),
$$

а дисперсия отклонения $\varepsilon\left(t_{0}+\Theta\right)$ выражается формулой

$$
D_{\varepsilon}=\sigma^{2}\left[1-\rho^{2}(\Theta)\right] .
$$

Поскольку $\rho(\Theta)$ - фактически нормированная автокорреляционная функция процесса $x(t)$, которая уменьшается от значения $\rho(\Theta=0)=1$ до $\rho(\Theta=\infty)=0$, то дисперсия $D_{\varepsilon}$ ошибки прогноза по одной точке $x\left(t_{0}\right)$ увеличивается от нуля (при $\Theta=0)$ до $\sigma^{2}$ (при $\left.\Theta=\infty\right)$.

Если $\Delta t-$ интервал дискретизации процесса $x(t)$, то удобно представить дисперсию ошибки прогноза в виде

$$
D_{\varepsilon}=\sigma^{2}\left[1-\rho^{2}(\Delta t)\right] .
$$

Последнее выражение указывает на то, что случайность появления значимой ошибки прогноза при малом $\Delta t$ обусловлена случайностью значимого отклонения $x\left(t_{0}+\Delta t\right)$ от математического ожидания $m$ в стационарном процессе $x(t)$.

Коррекция модели прогнозирования Математическая модель (1) экстраполяции 2-го порядка используется, главным образом, для построения прогностических фильтров для стационарных случайных сигналов. В таких моделях каждое спрогнозированное значение $x\left(t_{0}+\Theta\right)$ ближе к математическому ожиданию $m$, чем значение предыдущее $x\left(t_{0}\right)$ Естественно, что и дисперсия $D_{\varepsilon}$ отклонения $\hat{x}\left(t_{0}+\Theta\right)$ от фрактического значения $x\left(t_{0}+\Theta\right)$ меньше, чем дисперсия $\sigma^{2}$ исходного процесса. Это является ограничением по применению модели прогнозирования (1) по отношению к случайным выбросам, поскольку последние попросту фильтруются.

Однако, если выбросы провоцируются нарушениями стационарности по математическому ожиданию (в интервале дискретизации $\Delta t$ ), то имеет смысл скорректировать спрогнозирован- ное значение на величину априори известного параметра нестационарности $\Delta$ случайного процесса, учитывающего смещение его математического ожидания по отношению к его моде и медиане $[7,9,11]$ :

$$
\left\{\begin{array}{lll}
m_{x}=m, & \text { если } & x(t) \in \omega_{0}, \\
m_{x}=m+\Delta, & \text { если } & x(t) \in \bar{\omega}, \Delta=\mathrm{const},
\end{array}\right.
$$

где $\boldsymbol{\Delta}$ - параметр смещения (параметр нестационарности по математическому ожиданию); или на величину оценки его среднего значения $\overline{m_{\Delta}}$ выражение

$$
\bar{m}_{\Delta}=\left(\sum_{i=1}^{k} \bar{u}_{i} \cdot \hat{p}_{i}\right)-m .
$$

Учет такой коррекции представлен математической моделью

$$
\hat{x}\left(t_{0}+\Theta\right)=m+\Delta+\rho(\Theta)\left[x\left(t_{0}\right)-m\right] .
$$

Естественно, что дисперсия ошибки прогноза возрастает, и будет определяться выражением

$$
D_{\varepsilon k}=\left(\sigma^{2}+\Delta^{2}\right)\left[1-\rho^{2}(\Theta)\right] .
$$

Модель прогнозирования (6) имеет смещение $\Delta$ и дополнительную дисперсию ошибки прогнозирования

$$
D_{\Delta}=\Delta^{2}\left[1-\rho^{2}(\Theta)\right]
$$

Дополнительная дисперсия $D_{\Delta}$ тем меньше, чем меньше интервал прогнозирования $\Theta$. Выражение $(7,8)$ показывает, что дисперсия $D_{\Delta}$ максимальна и равна $\Delta^{2}$, если $\Theta=\infty$. Отсюда следует, что при малых значениях времени $\Theta$ выигрыш от коррекции (по выражению 6) не снижается из-за появления составляющей $D_{\Delta}$ в дисперсии ошибки прогнозирования.

Можно показать, что прогнозирование выбросов, обусловленных локальной нестационарностью, процесса загрязнения тем эфффективнее, чем больше компонент учитывается при контроле. Необходимо осуществлять прогнозирование выбросов не по одной, а по всем взаимно коррелированным компонентам процесса загрязнения, используя их как составляющие общего векторного процесса

$$
\bar{x}(t)=\left(x_{1}(t), \ldots x_{n}(t)\right),
$$

где $\left(x_{1}(t), \ldots x_{n}(t)\right)$ - случайные процессы изменения во времени компонент $\left(x_{1}, \ldots x_{n}\right)$. Учет при прогнозировании составляющих вектора $\bar{x}(t)$, статистически эквивалентен увеличению объема выборки значений $\left(x_{1}\left(t_{0}\right), \ldots x_{n}\left(t_{0}\right)\right.$, повышая структурно-информационную избыточность и увеличивая, в конечном счете, достоверность контроля. Общая процедура такого контроля может быть построена на применении операций суммирования центрированных и нормированных значений $\dot{x}_{1}(t), \ldots \dot{x}_{n}(t)$ и тестирования полученной суммы по критерию Стьюдента $[10,11]$. 
Параметрический тест на значимость нарушений стационарности.

Такой тест реализует процедуру проверки статистических гипотез и включает следующие этапы:

1. Выбирается уровень значимости $\alpha$ (риск контроля первого рода).

2. Задается статистическая модель отклонений экстраполированных значений $\left(\hat{x}_{1}\left(t_{0}\right), \ldots \hat{x}_{n}\left(t_{0}\right)\right.$ вектора $\bar{x}(t)$ от своих математических ожиданий $m_{1}, \ldots m_{n}$ как $n$-мерная нормально распределенная случайная величина $\bar{\Delta}$ с вектором-столбцом средних значений

$$
m=\left|\begin{array}{c}
m_{1} \\
m_{2} \\
\cdots \\
m_{n}
\end{array}\right|,
$$

и диагональной дисперсионной матрицей

$$
S=\left|\begin{array}{cccc}
\sigma^{2}{ }_{1} & 0 & \ldots & 0 \\
0 & \sigma^{2}{ }_{2} & \ldots & 0 \\
. & . & . & . \\
0 & 0 & \ldots & \sigma_{n}^{2}
\end{array}\right| .
$$

Плотность распределения вектора отклонений $\bar{\Delta}$ имеет вид

$$
\begin{gathered}
f(\bar{\Delta})=(2 \pi)^{-\frac{n}{2}}|S|^{-\frac{1}{2}} \times \\
\times \exp \left[-0,5(\bar{\Delta}-m) S^{-1}(\bar{\Delta}-m)\right] .
\end{gathered}
$$

3. Формулируются нулевая и альтернативная гипотезы

$$
\begin{aligned}
& H_{0}=\bar{\Delta}=0, \\
& H_{1}=\bar{\Delta} \neq 0 .
\end{aligned}
$$

4. Выбирается критериальная статистика

$$
t_{n-1}=n^{1 / 2} \cdot \sum_{i=1}^{n}\left[\frac{\hat{x_{i}}\left(t_{0}\right)-m_{i}}{\sigma_{i}}\right]
$$

в которой суммируются центрированные по математическому ожиданию и нормированные по дисперсии отклонения

$$
{\stackrel{\circ}{x_{i}}}_{(}\left(t_{0}\right) \sim \operatorname{NORM}(0,1), i=1, \bar{n} .
$$

5. Выбирается критическая область $\bar{\omega}$ для статистики $t$

$$
\bar{\omega}=\left(t_{n-1, \alpha}, \infty\right)
$$

6. Принимают одно из двух решений

$$
\begin{cases}\gamma_{0}: & \text { не отвергнуть гипотезу } \mathrm{H}_{0}, \text { если } t \neq \bar{\omega} \\ \gamma_{1}: & \text { отвергнуть гипотезу } \mathrm{H}_{0} \text { (не отвергнуть } \\ & \text { гипотезу } \left.\mathrm{H}_{1}\right), \text { если } t \neq \bar{\omega}\end{cases}
$$

Такой алгоритм тестирования реализует процедуру проверки статистических гипотез и позволяет оценить значимость нарушений стационарности.

\section{Выводы}

1. Использование скорректированной, в сторону увеличения математического ожидания, модели экстраполяции 2-го порядка, снижает риск контроля 2-го порядка $\beta$, при параметрическом тестировании результатов многомерной экстраполяции.

2. Уменьшение риска контроля $\beta$ повышает мощность правила принятия решений и делает применение описанной скорректированной процедуры экстраполяции статистически обоснованным и более предпочтительным по отношению к другим процедурам экстраполяции.

3. Целесообразно при планировании и прогнозировании контроля выбросов и сбросов энергоемких предприятий использовать тестирование с учетом правила принятия решений на основе разработанного критерия, что обеспечивает заданную достоверность.

\section{Литература}

1. Гук Ю.Б. Теория надежности в электроэнергетике/ Ю.Б. Гук / Л.: Энергоиздат, 1990. $-208 \mathrm{c}$

2. Филипов М. В. Подходы к оценке остаточного ресурса технических объектов [Текст] / М.В.Филипов, А.С. Фурсов, В.В. Клюев / К.: Контроль. Диагностика. - 2006. - №8 (98). - С. 6 -16.

3. Бондаренко В.Е. Оптимизация системы информационных показателей качества трансформаторного, для технического эксплуатационного контроля маслонаполненного энергетического оборудования / В.Е. Бондаренко, О.В. Шутенко / Х.:Інформаційно-керуючі системи на залізничному транспорті. - №2. - 2003. - С.46 - 50.

4. Надежность и эффективность в технике: Справочник в 10 т. / Ред сонет: В.С. Авдуевский (предс.) и др. - М.: Машиностроение, 1987. - Т.9: Техническая діагностика. Под. ред. В.В. Клюева, Г.П.Пархоменко. - 352 с.

5. Малайчук В.П. Обработка многомерных нестационарных случайных пространственновременных рядов в задачах мониторинга. [Текст] / В.П. Малайчук, А.В. Мозговой // Методи та прилади контролю якості. - Івано-Франківськ. - 2005. - № 15. - с.90 - 93.

6. Щапов П. Ф. Планирование профилактического контроля маслонаполненного енергетического оборудования для выявления процессов старения с заданной достоверностью принятия решений // X.: Электротехніка та електромеханіка. - 2005. - №3. - С. 65 - 68.

7. Щапов П. Ф. Многоканальная термометрия при прогнозировании сосотояний термодинамических систем // сник НТУ «ХПІ»: Зб. Наук. праць. 
Тематичний випуск: Автоматика та приладобудування. - 2003. - №7. - Т.3. - С.155 - 160.

8. Любимова Н.А. Коррекция прогнозирующих процедур при контроле загрязняющих выбросов энеергетических предприятий / Любимова Н.А. // Технологический аудит и резервы производства - Х. - 2014. - №3(17). - С. 42 - 45.

9. Пат. 60390 Украина, МПК G06G7/30 Цифрровий оптимальний екстраполятор нестаціонарного траффіку комп'ютерних мереж / Гузій М. М. , Андреєв О. В., Ігнатов В. О., Андрєєв В. ; заявник та патентовласник Національний авіаційний університет. - №201006549; заявл. 28.05.2010; опубл. 25.06.2011. - Бюл. №12. - 2011 р.

10. Вентцель Е.С. Теория случайных процессов и ее инженерные приложения / Е.С. Вентцель, Л.А. Овчаров - М.: Высшая школа. -2000 $-383 \mathrm{c}$.

11. Поллард Дж. Справочник по вычислительным методам статистики / Пер. с англ. В.С.Занадворова; под ред. и с предис. Е.М. Четыркина. - М.: Финансы и статистика. - 1982. - 344c., илл.

\section{Reference}

1. Guk Yu.B. Teoriya nadezhnosti v elektroenergetike/ Yu.B.Guk / L.: Energoizdat, 1990. - 208 s.

2. Filipov M. V. Podkhody $k$ otsenke ostatochnogo resursa tekhnicheskikh obektov [Tekst] / M.V.Filipov, A.S. Fursov, V.V. Klyuev / K.: Kontrol. Diagnostika. - 2006. - №8 (98). - S. 6 - 16.

3. Bondarenko V.Ye. Optimizatsiya sistemy informatsionnykh pokazateley kachestva transformatornogo, dlya tekhnicheskogo ekspluatatsionnogo kontrolya maslonapolnennogo energeticheskogo oborudovaniya / V.Ye. Bondarenko, O.V. Shutenko / Kh.:Informatsiyno-keruyuchi sistemi na zaliznichnomu transporti. - №2. - 2003. - S.46 - 50.

4. Nadezhnost i effektivnost $v$ tekhnike: Spravochnik v 10 t. / Red sonet: V.S. Avduevskiy (preds.) i dr. - M.: Mashinostroenie, 1987. - T.9: Tekhnicheskaya diagnostika. Pod. red. V.V. Klyueva, G.P. Parkhomenko. - 352 s.

5. Malaychuk V.P. Obrabotka mnogomernykh nestatsionarnykh sluchaynykh prostranstvennovremennykh ryadov $v$ zadachakh monitoringa. [Tekst] / V.P. Malaychuk, A.V. Mozgovoy // Metodi ta priladi kontrolyu yakosti. - Ivano-Frankivsk. - 2005. - № 15. - s.90 - 93.

6. Shchapov P. F. Planirovanie profilakticheskogo kontrolya maslonapolnennogo energeticheskogo oborudovaniya dlya vyyavleniya protsessov stareniya $s$ zadannoy dostovernostyu prinyatiya resheniy // Kh.: Elektrotekhnika ta elektromekhanika. - 2005. - №3. - S. 65 - 68.

7. Shchapov P. F. Mnogokanalnaya termometriya pri prognozirovanii sosotoyaniy termodinamicheskikh sistem // snik NTU «KhPI»: Zb. Nauk. prats. Tematichniy vipusk: Avtomatika ta priladobuduvannya. - 2003. - №7. - T.3. - S.155 - 160.

8. Lyubymova N.A. Korrektsiya prognoziruyushchikh protsedur pri kontrole zagryaznyayushchikh vybrosov eneergeticheskikh predpriyatiy / Lyubimova N.A. // Tekhnologicheskiy audit i rezervy proizvodstva - Kh. - 2014. - №3(17). - S. 42 - 45.

9. Pat. 60390 Ukraina, MPK G06G7/30 Tsifroviy optimalniy ekstrapolyator nestatsionarnogo trafiku komp'yuternikh merezh / Guziy M. M., Andreev O. V., Ignatov V. O., Andre€v V.; zayavnik ta patentovlasnik Natsionalniy aviatsiyniy universitet. - № 201006549; zayavl. 28.05.2010; opubl. 25.06.2011. - Byul. №12. $-2011 \mathrm{r}$.

10. Venttsel Ye.S. Teoriya sluchaynykh protsessov i ee inzhenernye prilozheniya / Ye.S. Venttsel, L.A. Ovcharov - M.: Vysshaya shkola. $-2000-383$ s.

11. Pollard Dzh. Spravochnik po vychislitelnym metodam statistiki / Per. s angl. V.S.Zanadvorova; pod red. i s predis. Ye.M.Chetyrkina. - M.: Finansy i statistika. - 1982. - 344s., ill.

\title{
Аннотація
}

\section{План контролю димових газів енергопідприємств з урахуванням порушень стаціонарності викидів}

\author{
Н.О. Любимова, В.К. Пузік, Л.М. Пузік
}

Дане дослідження присвячене плануванню та прогнозуванню контролю димових газів крупних енергоємних підприємств. Поява випадкових технологічних порушень провокує випадковість викидів, порушення норм природокористування, економічні санкції та штрафи, призводить до занепаду навколишнього середовища. Розроблена математична модель планування i прогнозування екстремальних викидів у випадкових процесах забруднення димових газів енергетичних підприємств із корекцією на не стаціонарність процесів. Досліджена процедура параметричного тестування багатокомпонентного процесу забруднення при багато параметричному контролі із урахуванням його екстрапольованих значень. Використання скорегованої в бік збільшення математичного очикування, модулі екстраполяції 2-го порядку, знижує ризик контролю 2-го порядку $\beta$, при параметричному 
тестуванні результатів багатовимірної екстраполяції. Зменшення ризику контролю $\beta$ збільшує потужність правила прийняття рішення та робить застосування запропонованої скорегованої процедури екстраполяції статистично обґрунтованою та більш інформаційними по відношенню до інших процедур екстраполяції. Доцільно при плануванні та прогнозуванні контролю викидів та скидів енергоємних підприємств використовувати тестування 3 урахуванням правила прийняття рішень на основі розробленого критерію, який забезпечує задану достовірність. Оцінка стаціонарності та спектральних особливостей досліджуваних процесів забруднення дозволить зменшити апріорну невизначеність та дасть можливість удосконалити процедуру контролю димових газів. Вибір правила прийняття рішення на основі критерію, який забезпечує задану достовірність контролю, дозволить дослідити кореляційну пов'язаність контрольованого процесу та розробити статистично обґрунтований метод вибору інтервалу його дискретизації. Даний підхід гарантує мінімізацію тих його ризиків, які визначають рівень економічних втрат при появі порушень стаціонарності. Запропоновану модель контролю можливо використовувати при екологічному моніторингу викидів в атмосферу енергоємних підприємств

Ключові слова: екологія, модель, контроль, прогнозування, багатокомпонентне забруднення.

Abstract

\title{
Fuel gas control plan for energy enterprises taking into account emissions stability disorders
}

\author{
N.O. Lyubymova, V.K.Pusik, L.V. Pusik
}

This study focuses on planning and forecasting the control of flue gases of large energy-consuming enterprises. . The occurrence of random technological violations provokes randomness of emissions, violation of environmental standards, economic sanctions and fines, leading to environmental degradation.

A mathematical model of planning and forecasting extreme emissions in random processes of flue gas pollution of energy companies with a correction for non-stationarity of processes has been developed. A procedure for parametric testing of a multicomponent process of contamination in the case of multi-parameter control with regard to its extrapolated values is described. The use of an adjusted, in the direction of increasing the mathematical expectation, a model of extrapolation of the 2 nd order, reduces the risk of control of the 2 nd order, with parametric testing of the results of multidimensional extrapolation. Reducing the risk of control increases the power of the decision rule and makes the application of the described adjusted extrapolation procedure statistically justified and preferable to other extrapolation procedures. It is advisable when planning and predicting the control of emissions and discharges of energy-intensive enterprises to use testing based on the decision rule based on the developed criterion, which ensures the specified accuracy. Evaluation of the stationarity and spectral features of the contamination processes under study will reduce the a priori uncertainty and provide an opportunity to improve the flue gas control procedure. The choice of the decision rule based on the criterion that ensures the specified accuracy of the control will allow to investigate the correlation connectivity of the monitored process and to develop a statistically based method for choosing the interval of its discretization. This approach ensures the minimization of those risks that determine the level of economic losses in case of violations of stationarity. The proposed control model can be used for environmental monitoring of emissions of energy-intensive enterprises into the atmosphere.

Keywords: ecology, model, control, forecasting, multicomponent, pollution

\section{Бібліографічне посилання/ Bibliography citation: Harvard}

Lyubymova, N., Pusik,V., and Pusik, L. (2019). Fuel gas control plan for energy enterprises taking into account emissions stability disorders. Engineering of nature management, (3(13), pp. 62 - 66.

Подано до редакції / Received: 13.03.2019 